\title{
Brain Tumor Texture Analysis - Using Wavelets and Fractals
}

\author{
Tuhin Utsab Paul ${ }^{1}$, Aninda Ghosh ${ }^{1}$, Samir Kumar Bandhyopadhyay ${ }^{2, *}$ \\ ${ }^{1}$ Department of Electronics and Communication, Institute of Engineering and Management, Kolkata, India \\ ${ }^{2}$ Department of Computer Science and Engineering, University of Calcutta, Kolkata, India
}

Email address:

2.tuhin.kol@gmail.com (T. U. Paul), anindaghosh63@gmail.com (A. Ghosh), 1954samir@gmail.com (S. K. Bandhyopadhyay)

${ }^{*}$ Corresponding author

\section{To cite this article:}

Tuhin Utsab Paul, Aninda Ghosh, Samir Kumar Bandhyopadhyay. Brain Tumor Texture Analysis - Using Wavelets and Fractals. International Journal of Medical Imaging. Vol. 4, No. 4, 2016, pp. 23-31. doi: 10.11648/j.ijmi.20160404.11

Received: July 12, 2016; Accepted: July 26, 2016; Published: August 15, 2016

\begin{abstract}
Brain tumor segmentation is quite popular area of research but detection of its surface texture is challenging for researchers. Normally, MRI datasets have very low resolution. This paper utilizes image enhancement technique based on wavelet. It is used to scale the low resolution image to a suitable resolution without loss. Secondly the proposed method is focused on implementation of a trained classifier using features: fractal dimension, fractal area, and wavelet average to classify type of texture present in brain tumor.
\end{abstract}

Keywords: Wavelet Transform, Fractal Analysis, Image Classification, Feature Extraction, Texture Analysis, Image Processing

\section{Introduction}

Automatic image analysis has a lot of scope for implementation, from image classification to image retrieval. Semantic gap problem [1, 2], which corresponds to the alteration between the user image perception and automatic extracted features. An important aspect of feature extraction task is to obtain a set of features (i.e. a feature vector) for representing the visual content of an image. In many applications texture features are used for maintaining orthogonal nature among different classes. On the other hand, it can be effectively implemented to address the semantic gap problem. As a result, research on texture classification is focused on improving the distinguish-ability of the algorithm.

In the paper the problem of identifying the type of texture in tumor of brain from MRI is addressed. Segmentation technique is utilized to visually extract the position and size of the tumor. Wavelet transform is established as a very efficient tool for analyzing an image's frequency components in a localized manner. The popular SFTA algorithm, is used along with the features extracted from wavelet transform.

The input MR Image is first subdivided in small blocks of pixel. Next, texture of each sub block is obtained in order to identify the texture of the tumor. Multi resolution wavelet transform is implemented to get the details of input image including energy and frequency details. The feature vector thus obtained is easily achievable in terms of complexity. The feature vector is $1 \times 26$ array consisting of multi resolution wavelet detail of different wavelet families.

\section{Related Works}

In image processing textural information plays a key role to identify the type of object present in the images. It dominates in the field of remote sensing, quality control \& medical imaging due to its close relation to the underlying semantics.

Texture features captures the granularity and the repetitive pattern in the image. From statistical point of view texture information can be termed as fractal whose Area, Mean, Dimension can be accepted as Fractals features. Fractals [3] are small pictorial patterns those tend to repeat in a textured surface.

Another widely accepted approach is to use grayscale cooccurrence matrices (GLCM) by counting the number of occurrences of the gray levels at a given displacement and 
angle. Statistical quantities such as contrast, energy, entropy are computed from the GLCM to obtain the texture features as proposed by Haralick et al.

Filter Bank [4, 5] approach is a widely accepted method for texture classification and segmentation. Gabor filters is among the popular filter banks known for its invariance with respect to scale, rotation and displacement. Where $\theta$ is the filter orientation, $\sigma$ the standard deviation and $\lambda$ the wavelength of the sinusoid,

Equation shows the formal representation of the Gabor filter,

$$
\begin{gathered}
g(x, y)=\left(1 / 2 \pi \sigma_{x} \sigma_{y}\right)\left(-\frac{1}{2} A+\frac{2 \pi i R_{1}}{\lambda}\right) \\
A=\left(\frac{R_{1}^{2}}{\sigma_{x}^{2}}+\frac{R_{2}^{2}}{\sigma_{y}^{2}}\right) \&\left[\begin{array}{l}
R_{1} \\
R_{2}
\end{array}\right]=\left[\begin{array}{cc}
\cos \theta & \sin \theta \\
-\sin \theta & \cos \theta
\end{array}\right]
\end{gathered}
$$

The pixel position is given by $\mathrm{x}, \mathrm{y}$.

Fractal dimension measurements can be used to estimate and quantify the complexity of the shape or texture of objects. The most common is the Hausdorff's dimension. An object with a Euclidean Dimension E, Hausdorff's fractal dimension $\mathrm{D}_{0}$ computed by the following equation:

$$
\mathrm{D}_{0}=\lim _{\mathrm{e} \rightarrow 0} \frac{\log \mathrm{N}(\mathrm{e})}{\log \mathrm{e}^{-1}}
$$

where $N(E)$ is the counting of hyper-cubes of dimension $E$ and length that fill the object.

Hausdorff's dimension [7, 8] is an important feature describing the fractal structure. The algorithm for obtaining the dimension value can be described as follows, if an object is described using a binary image $\mathrm{I}_{\mathrm{b}}$ then an approximate Dimension value van be calculated using box counting algorithm. The image is divide into small sub matrix of size e $x$ e. By varying the size, it is possible to generate an approximated straight line curve using $\frac{\log N(e)}{\log e^{-1}}$. The slope of the line gives Hausdorff dimension.

Another prior art is FFS [9] (Fast Fractal Stack) in which the fractal dimension is computed from a set of binary images obtained from the input grayscale image using the binary stack decomposition algorithm. The image is decomposed by applying successive operation using threshold value. Depending on the number of threshold value, image forms a binary image stack. The main disadvantage of this method since threshold intensities are chosen at an equal interval hence a lot of information gets lost during the operation.

The above algorithm proves to have an efficiency of $76 \%$ when implemented with a Knn classifier [10, 11] and $80 \%$ when trained with a discriminant based classifier. It is time consuming algorithm. For achieving higher classifier efficiency number of operation needs to be increased, hence there is a tradeoff between the classifier efficiency and the speed of the algorithm.

The thresholding using multilevel Otsu algorithm improves the intra class variance between the binary stack images is least.

\section{Proposed Method}

Wavelet Decomposition technique to the input image is firstly applying for obtaining the feature based on the decomposition. Secondly applying multilevel Otsu algorithm for finding threshold of the input image and then implementing box counting algorithm to obtain the features from it. The features obtained from both algorithms will serve as the feature set for the classifier. The trained classifier will then utilize it to predict texture of the new image.

Wavelet proves to be a very suitable and efficient method to obtain the localized frequency information of any signal. Previously frequency details were retrieved using Fast Fourier Transform. On the other hand, Wavelet based transform gives the localized information about the frequency content as feature.

Wavelet transform over the image generates two kind of features:

(i) Approximated image $\left(\mathrm{A}_{\mathrm{i}}\right.$, where i denotes the level of transform $)$

(ii) Frequency Detail (also termed as detail in the rest of the paper) of the input image. Depending on the level of transform the features that are obtained increases in a tree form. $\left(D_{i}\right.$, where i denotes the level of transform $)$

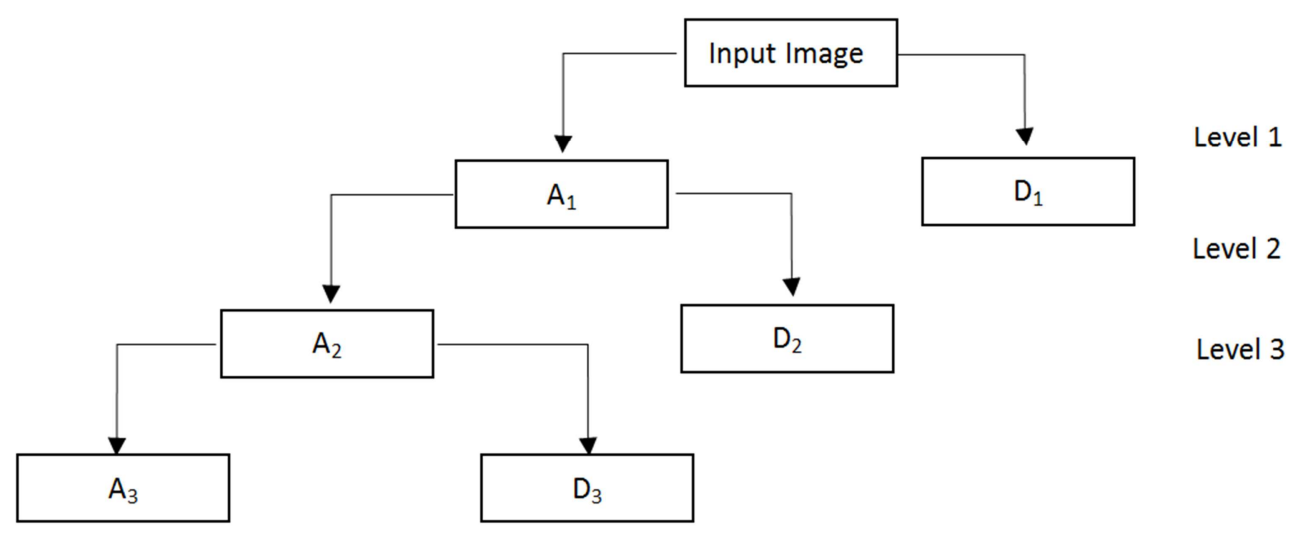

Fig. 1. Frequency guided level wise decomposing of image. 
Input image $=\mathrm{A}_{3}+\mathrm{D}_{3}+\mathrm{D}_{2}+\mathrm{D}_{1}$

When the images are decomposed into further levels then approximate image (Ai) gets smoother and holds the information about low pass filter applied over the initial image. On the other hand, frequency domain details of the image (Di) preserves the high pass filter information.

These values use as a set for classifying texture. The decomposition Algorithm finds the Energy values and Detail values calculated using different types of wavelet packets. The Wavelet families are used for feature extraction (i) Haar (ii) Daubechious (iii) Bior (iv) Coiflet.

The following algorithm is used to find out feature vector using wavelet transform:

I. Load the image

II. Convert it into gray scale image

III. For the converted image for each wavelet 5 levels of detail are obtained (details need to be specified)

IV. Store all the 20 detail values and 4 Energy values corresponding to the 4 wavelet families

V. First set of feature vectors are stored.

Energy and Detail of the input image are obtained using these equations.

$$
\begin{gathered}
E_{\text {detail }}=\sqrt{d_{j}^{H}+d_{j}^{v}+d_{j}^{D}} \\
E_{\text {approx }}=\sqrt{\left(a_{j}\right)^{2}} \\
E_{\text {ratio }}=E_{\text {detail }} / E_{\text {approx }}
\end{gathered}
$$

After the first set of features are obtained, second set of features are extracted from the fractal analysis. This Algorithm incorporates two basic algorithm (i) Multilevel Otsu Algorithm [12] (ii) Bounding box technique to obtain the fractal dimension [3]

a) Multilevel Otsu Algorithm

The Otsu algorithm takes a grayscale image $\mathrm{I}(\mathrm{x}, \mathrm{y})$ as an input and calculate the user defined number of threshold points such that the intra class variance becomes minimum. It incorporates the gray level distribution function to generate the threshold levels. When the threshold points $(\mathrm{T})$ are obtained the next task is to generate the binary images using two threshold segmentation levels from the equation.

$$
\mathrm{I}_{\mathrm{b}}(\mathrm{x}, \mathrm{y})=\left\{\begin{array}{l}
1 \text { if } t_{1}<I(x, y) \leq t_{u} \\
0, \text { otherwise }
\end{array}\right.
$$

where $t_{1}$ and $t_{u}$ denote, respectively, lower and upper threshold values. The set of binary images is obtained by applying the two threshold segmentation to the input image using all pairs of contiguous thresholds from $\mathrm{T} \cup\{\mathrm{nl}\}$ and all pairs of thresholds $\{t, n l\}, t \in T$, where $n_{1}$ corresponds to the maximum possible gray level in I $(\mathrm{x}, \mathrm{y})$.

b) Box Counting Algorithm to compute the Fractal
Dimension, Area, Mean

After applying the Multilevel Otsu Algorithm to the input gray level image, the feature vector is constructed as the resulting binary images' size, mean gray level and boundaries' fractal dimension. The regions' boundaries of a binary image $\mathrm{I}_{\mathrm{b}}(\mathrm{x}, \mathrm{y})$ denoted by border image $\Delta(\mathrm{x}, \mathrm{y})$ and computed as follows:

$$
\Delta(x, y)=\left\{\begin{array}{l}
1 \text { if } \exists\left(x^{\prime}, y^{\prime}\right) \in N_{8}[(x, y)]: \\
I_{b}\left(x^{\prime}, y^{\prime}\right)=0 \wedge \\
I_{b}(x, y)=1 \\
0, \text { otherwise. }
\end{array}\right.
$$

where $\mathrm{N} 8[(\mathrm{x}, \mathrm{y})]$ denotes the set of pixels that are 8connected to $(\mathrm{x}, \mathrm{y}) . \Delta(\mathrm{x}, \mathrm{y})$ has value 1 if the pixel at position $(\mathrm{x}, \mathrm{y})$ in the corresponding binary image $\mathrm{I}_{\mathrm{b}}(\mathrm{x}, \mathrm{y})$ has the value 1 and having at least one neighboring pixel with value 0 . Otherwise, $\Delta(\mathrm{x}, \mathrm{y})$ takes the value 0 . Hence, one can realize that the resulting borders are one-pixel wide. Fractal Dimension are extracted using Box Counting algorithm in which the binary image is divided in to small grid and for each sub grid part the fractal dimension is stored given by the number of instance a border is found. Mean and Area of the fractals are computed without much computational power.

Having described the basic processing tools used in the algorithm, the complete explanation of the algorithm goes in the following way.

Algorithm can be decomposed in two stages:

A. Training a Discriminant classifier using some known datasets whose texture is known by the user.

B. Using the Discriminant classifier on the segmented tumor image for detecting the texture type.

\subsection{Training Phase Algorithm}

I. Input Image.

II. Feed the input image from the training datasets used.

III. Find level 1 detail of the Haar, Daubechies, BiOrthogonal, Coiflet wavelet transform.

IV. Using Multilevel Otsu algorithm to find two level threshold values.

V. Obtained Threshold values are used to generate two binary images.

VI. Fractal Dimensions are calculated using hausdorff method [7, 8].

VII. Using Fractal Dimension, Level 1 detail of the wavelets as features to identify the texture detail [3].

VIII.Repeat the previous steps until the features are stored for the whole dataset.

IX. Use the Feature matrix to train a Discriminant classifier with the labels as indicated in the following table. 
Table 1. Materials specifying texture classes.

\begin{tabular}{ll}
\hline Type & Class \\
\hline Floor & Slight Smooth \\
Marble & Very Smooth \\
Knit & Moderately Rough \\
Corduroy & Moderately Rough \\
Fur & Moderately Rough \\
Bark & Very Rough \\
Glass & Very Smooth \\
Brick & Slight Rough \\
Wall & Slight Rough \\
Upholstery & Slight Rough \\
Plaid & Slight Rough \\
Pebbles & Very Rough \\
Wallpaper & Slight Rough \\
Wood & Very Rough \\
Water & Slight Smooth \\
Granite & Moderately Smooth \\
Carpet & Moderately Rough \\
\hline
\end{tabular}

Table 2. Level 1 texture details obtained using OTSU Algorithm.

\begin{tabular}{llll}
\hline \multirow{2}{*}{ Class } & \multicolumn{3}{l}{ Feature values } \\
\cline { 2 - 4 } & Mean Level 1 & Area Level 1 & Dimension Level 1 \\
\hline Very Smooth (0\% Rough 100\% Smooth) & 1.726699823 & 79.36368951 & 82103 \\
Moderately Smooth (20\% Rough 80\% Smooth) & 1.7088903 & 83.24563 & 56231 \\
Slight Smooth (40\% Rough 60\% Smooth) & 1.677946534 & 114.1885945 & 59445 \\
Slight Rough (60\% Rough 40\% Smooth) & 1.3475866482 & 87.13088195 & 10987 \\
Moderately Rough (80\% Rough 20\% Smooth) & 1.712092195 & 75.91502644 & 68080 \\
Very Rough (100\% Rough 0\% Smooth) & 1.711876879 & 110.1846481 & 77710 \\
\hline
\end{tabular}

Table 3. Level 2 texture details obtained using OTSU Algorithm.

\begin{tabular}{llll}
\hline \multirow{2}{*}{ Class } & Feature values & & Dimension Level 2 \\
\cline { 2 - 4 } & Mean Level 2 & Area Level 2 & 81010 \\
\hline Very Smooth (0\% Rough 100\% Smooth) & 1.726953297 & 104.7969757 & 75092 \\
Moderately Smooth (20\% Rough 80\% Smooth) & 1.79839231 & 102.23231 & 19180 \\
Slight Smooth (40\% Rough 60\% Smooth) & 1.479879362 & 104.3540146 & 129215 \\
Slight Rough (60\% Rough 40\% Smooth) & 1.79390182 & 52.06027164 & 72014 \\
Moderately Rough (80\% Rough 20\% Smooth) & 1.721312591 & 77.54282501 & 45940 \\
Very Rough (100\% Rough 0\% Smooth) & 1.622193522 & 98.09344798 & \\
\hline
\end{tabular}

Table 4. Level 1 texture details obtained using Haar Wavelet.

\begin{tabular}{lll}
\hline \multirow{2}{*}{ Class } & Feature values & Haar Detail Level 1 \\
\cline { 2 - 3 } & Haar Average Level 1 & 2.685554273 \\
\hline Very Smooth (0\% Rough 100\% Smooth) & 97.31444573 & 0.56234244 \\
Moderately Smooth (20\% Rough 80\% Smooth) & 98.33424367 & 0.379219514 \\
Slight Smooth (40\% Rough 60\% Smooth) & 99.62078049 & 7.385489097 \\
Slight Rough (60\% Rough 40\% Smooth) & 92.6145109 & 2.242540978 \\
Moderately Rough (80\% Rough 20\% Smooth) & 97.75745902 & 0.88522254 \\
Very Rough (100\% Rough 0\% Smooth) & 99.11477746 & \\
\hline
\end{tabular}

Table 5. Level 1 texture details obtained using Daubechies Wavelet.

\begin{tabular}{lll}
\hline \multirow{2}{*}{ Class } & Feature values & \\
\cline { 2 - 3 } & Daubechies Average Level 1 & Daubechies Detail Level 1 \\
\hline Very Smooth (0\% Rough 100\% Smooth) & 98.73633881 & 1.263661195 \\
Moderately Smooth (20\% Rough 80\% Smooth) & 97.23242342 & 1.05243233 \\
Slight Smooth (40\% Rough 60\% Smooth) & 99.8322521 & 0.167747905 \\
Slight Rough (60\% Rough 40\% Smooth) & 94.91961194 & 5.080388064 \\
Moderately Rough (80\% Rough 20\% Smooth) & 98.73764835 & 1.262351652 \\
Very Rough (100\% Rough 0\% Smooth) & 99.44868289 & 0.551317111 \\
\hline
\end{tabular}


Table 6. Level 1 texture details obtained using Bi-Orthogonal Wavelet.

\begin{tabular}{lll}
\hline \multirow{2}{*}{ Class } & Feature values & \\
\cline { 2 - 3 } & Bi-Orthogonal Average Level 1 & Bi-Orthogonal Detail Level 1 \\
\hline Very Smooth (0\% Rough 100\% Smooth) & 97.18699987 & 2.81300013 \\
Moderately Smooth (20\% Rough 80\% Smooth) & 98.33423424 & 0.890334242 \\
Slight Smooth (40\% Rough 60\% Smooth) & 99.58879022 & 0.411209782 \\
Slight Rough (60\% Rough 40\% Smooth) & 92.51087882 & 7.489121185 \\
Moderately Rough (80\% Rough 20\% Smooth) & 97.67231902 & 2.327680976 \\
Very Rough (100\% Rough 0\% Smooth) & 99.04748648 & 0.952513519 \\
\hline
\end{tabular}

Table 7. Level 1 texture details obtained using Coiflet Wavelet.

\begin{tabular}{lll}
\hline \multirow{2}{*}{ Class } & Feature values & \\
\cline { 2 - 3 } & Coiflet Average Level 1 & Coiflet Detail Level 1 \\
\hline Very Smooth (0\% Rough 100\% Smooth) & 98.36831315 & 1.631686849 \\
Moderately Smooth (20\% Rough 80\% Smooth) & 97.4934932 & 1.152352324 \\
Slight Smooth (40\% Rough 60\% Smooth) & 99.78445554 & 0.215544459 \\
Slight Rough (60\% Rough 40\% Smooth) & 94.2230022 & 5.776997803 \\
Moderately Rough (80\% Rough 20\% Smooth) & 98.57000487 & 1.429995132 \\
Very Rough (100\% Rough 0\% Smooth) & 99.36600026 & 0.633999745 \\
\hline
\end{tabular}

\section{Variation of Texture in accordance of Mean level}

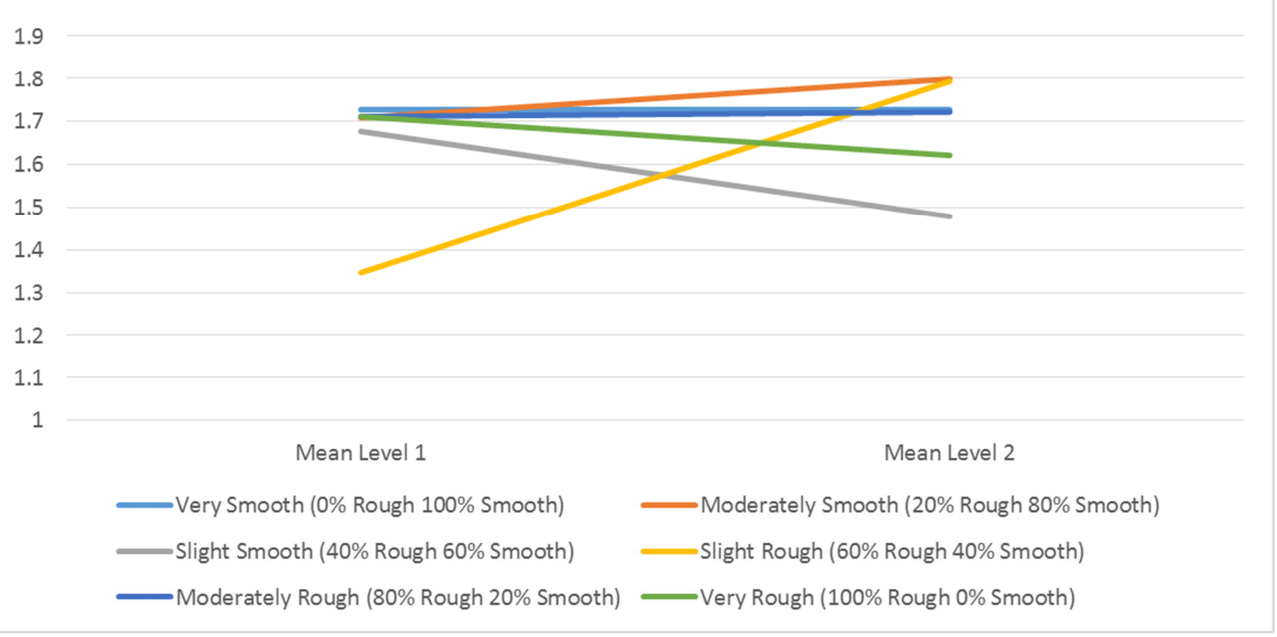

Fig. 2. Variation in Texture from Mean level 1 to level 2.

\section{Variation of Texture in accordance of Area level}

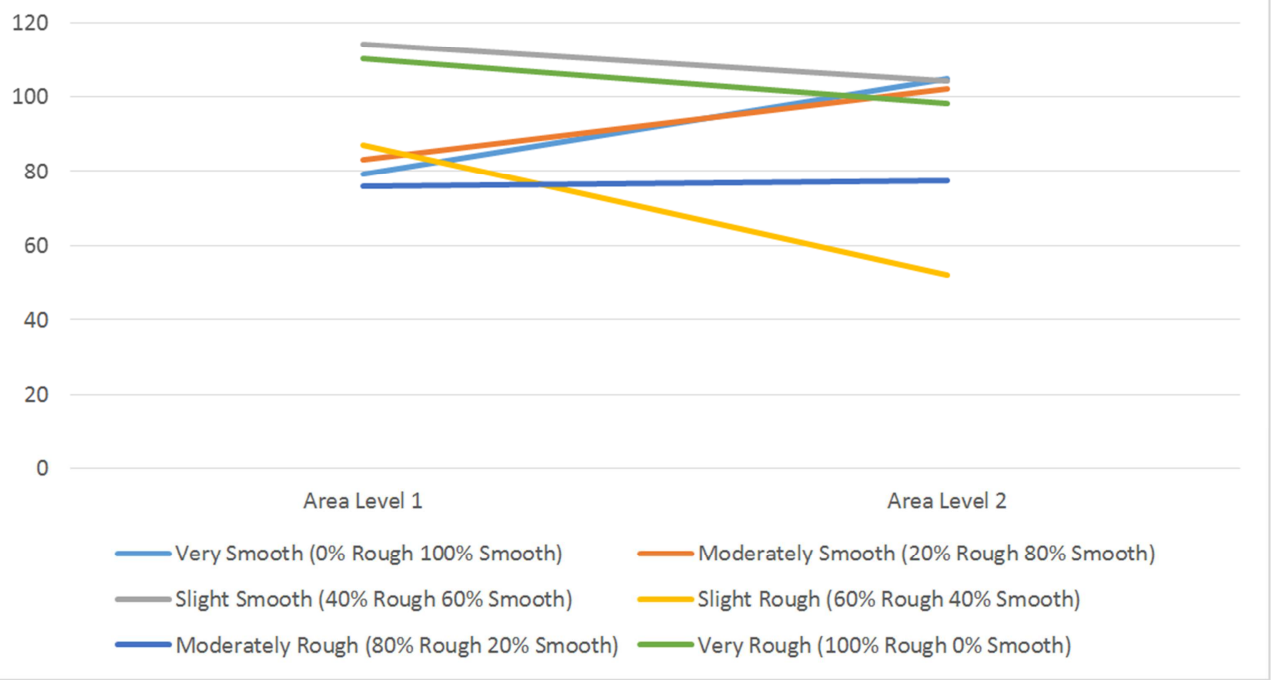

Fig. 3. Variation in Texture from Area level 1 to level 2. 


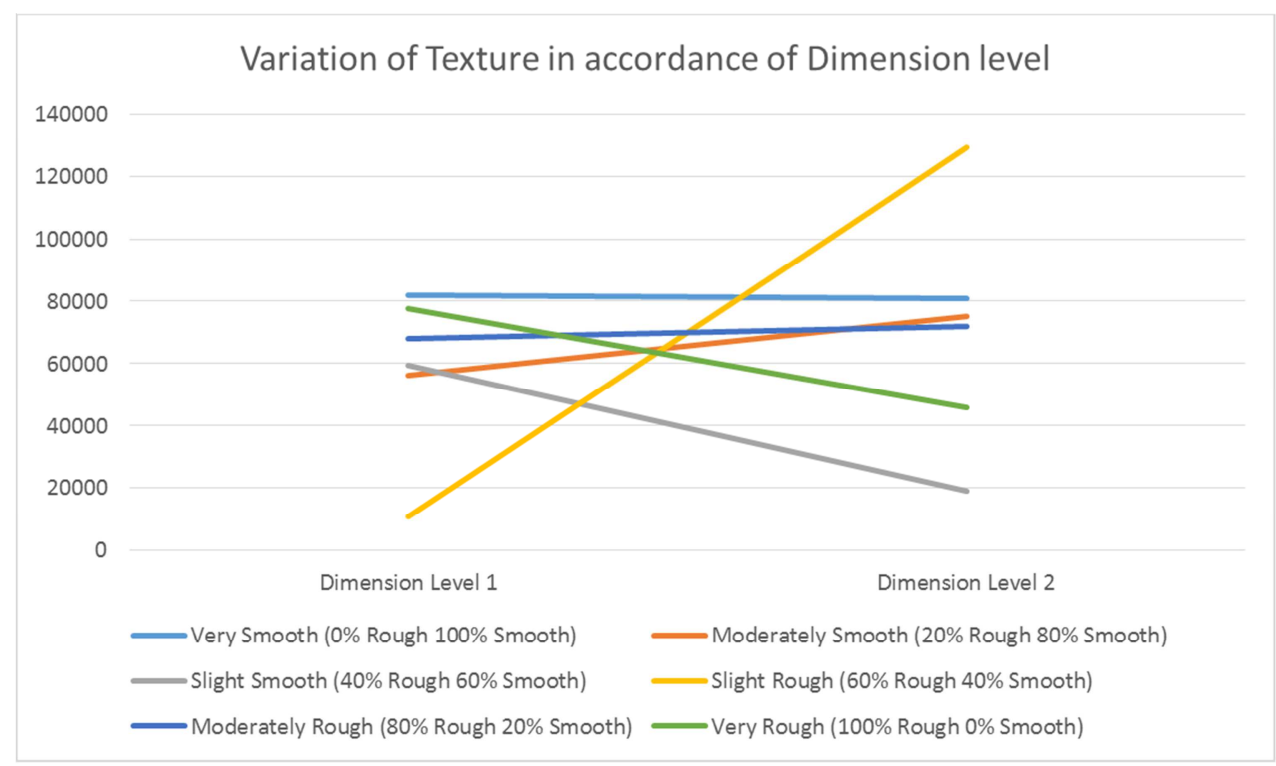

Fig. 4. Variation in Texture from Dimension level 1 to level 2.

Variation of Texture in Different Wavelet Average Values at level 1

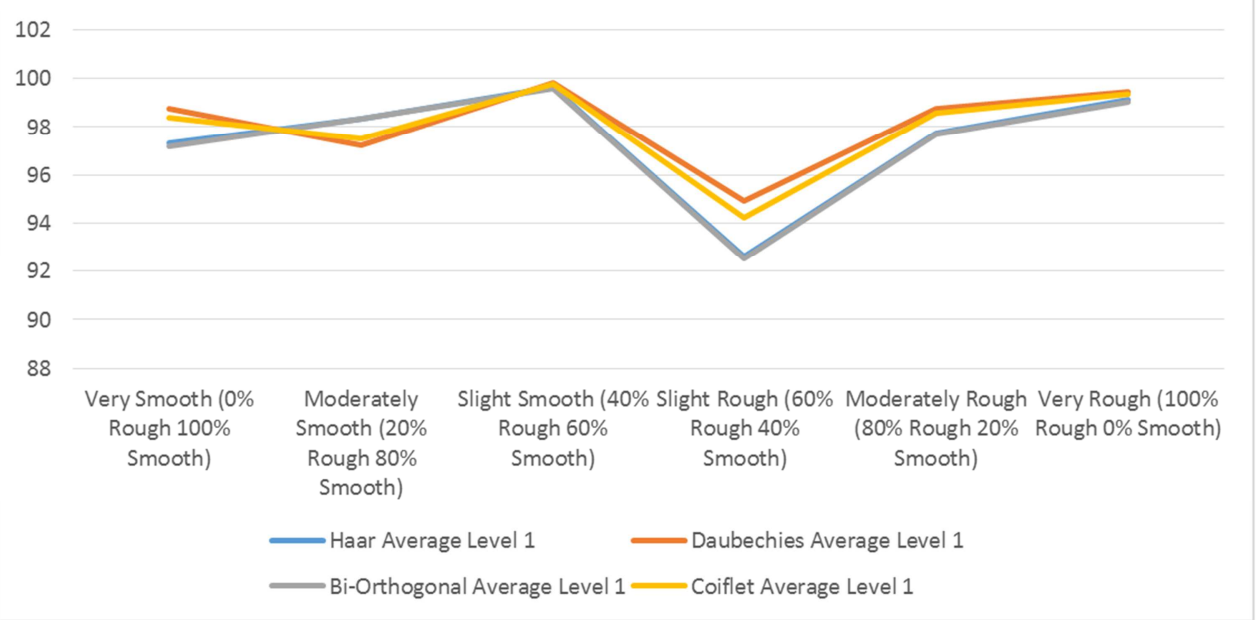

Fig. 5. Variation of Wavelet Average Values for Texture Classes.

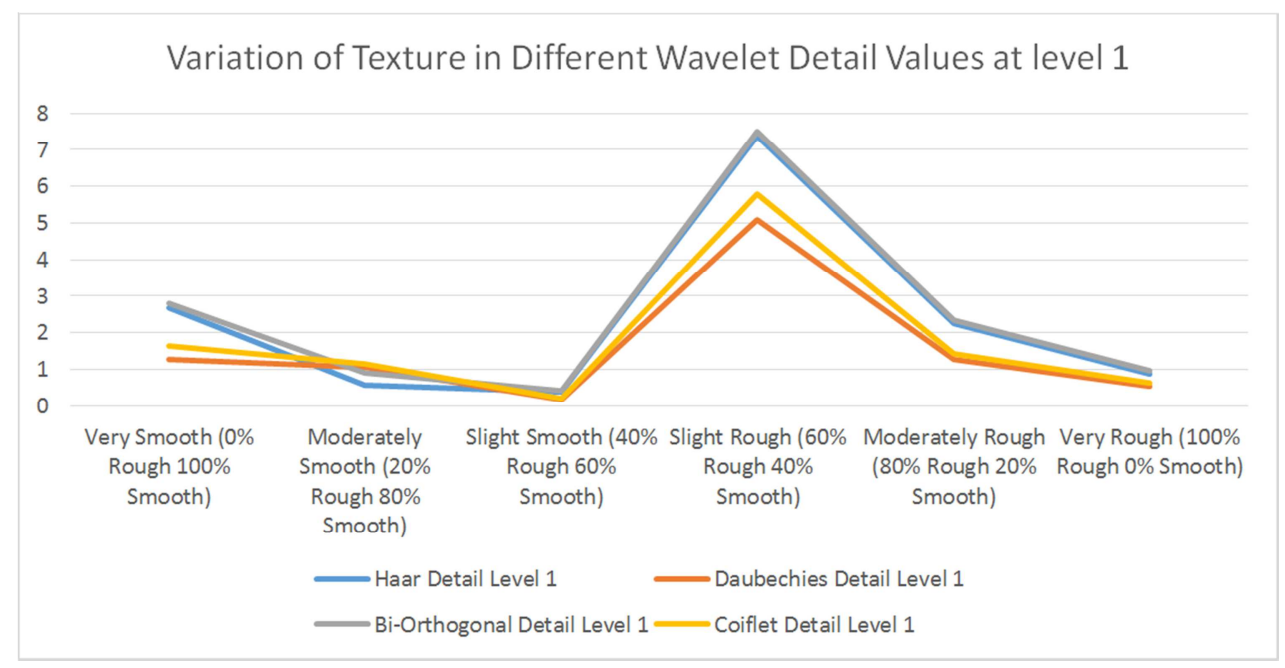

Fig. 6. Variation of Wavelet Detail Values for Texture Classes. 
As the experiments reveal the wavelet energy and details efficiently describe a texture pattern and forms a highly uncorrelated feature sets. More over the Fractal analysis algorithm not on describe the texture completely but also forms an orthogonal set of feature. As mentioned in the prior work [3] SFTA Algorithm was implemented which gave an amazing texture classification but with 4 threshold level. In our algorithm we reduced the number of threshold, but reducing the threshold points decreases the strength of the feature sets. So along with reduction in the threshold points we introduced the wavelet based features which not only improve the classification efficiency but also reduced the classification time.

\subsection{Deployment Phase Algorithm}

I. Input MR Image.

II. Equalize Histogram of the Image.

III. Separate out the Skull for Segmenting the Tumor from the brain.

a) Thresholding is implemented such that the binary image obtained so has least intra class variance.

b) Subtract the thresholded image to get the Skull less brain.

c) Divide the image in $64 * 64$ blocks

- For each block among 4096 divided blocks, Daubechies wavelet transform is implemented to segment out the tumor.

- Tumor is segmented using thresholding based on the wavelet based features and the coordinates are stored for further processing.

- Morphological Operations are performed so as to display the actual positions of the tumor as an image.

a) Finding the texture information for the blocks which have a part of the tumor is our area of interest, hence it reduces a ton of computational complexity.

b) For each block having a part of Tumor

- The size of the blocks is too small to find textures features so Wavelet based resolution enhancement is implemented.

- The image so obtained from each block is decomposed using Haar wavelet transform to generate Average Image, Horizontal Detail Image, Vertical Detail Image, Diagonal Detail Image.

- Each Image is resized 10 folds.

- All the new images so obtained are fed to inverse Haar wavelet transform to get the new resolution enhanced image.

- For each enhanced block

- Find level 1 detail of the Haar, Daubechies, BiOrthogonal, Coiflet wavelet transform.

- Using Multilevel Otsu algorithm to find two level threshold values.

- Obtained Threshold values are used to generate two binary images.

- Fractal Dimensions are calculated using hausdorff method.

- Using Fractal Dimension, Level 1 detail of the wavelets as features to identify the texture detail.

- The Features so obtained are fed to earlier trained Discriminant classifier and the output class is registered for each block having the tumor part.

- With the knowledge of the registered class for each block, a grayscale image is generated showing the texture details with the different grayscale levels.

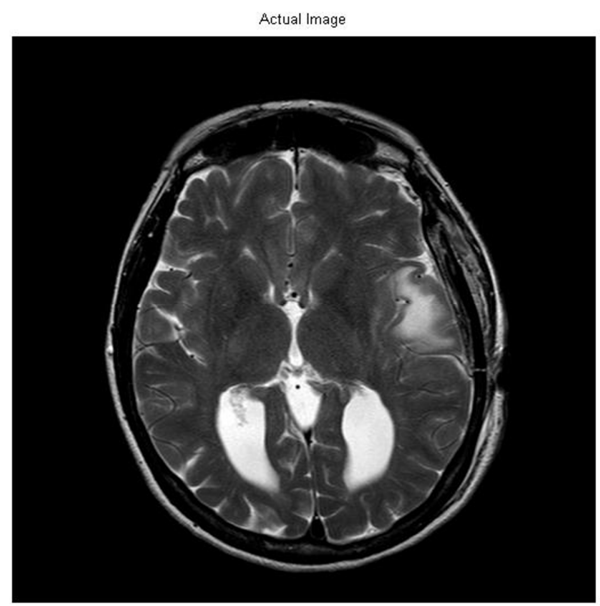

Fig. 7. Actual Image.

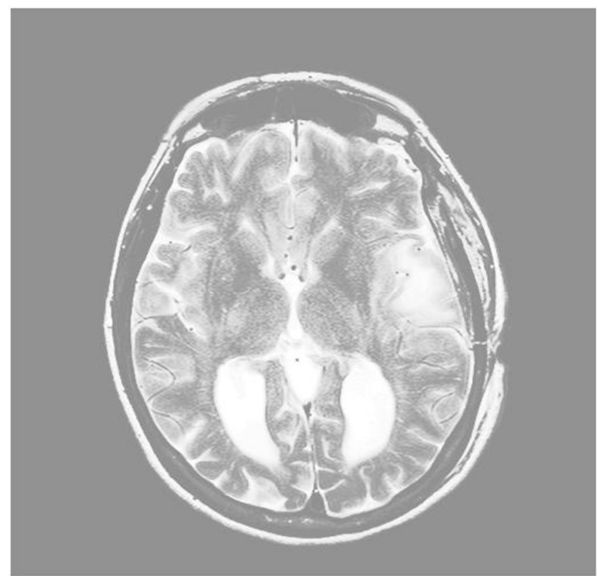

Fig. 8. Histogram Equalized

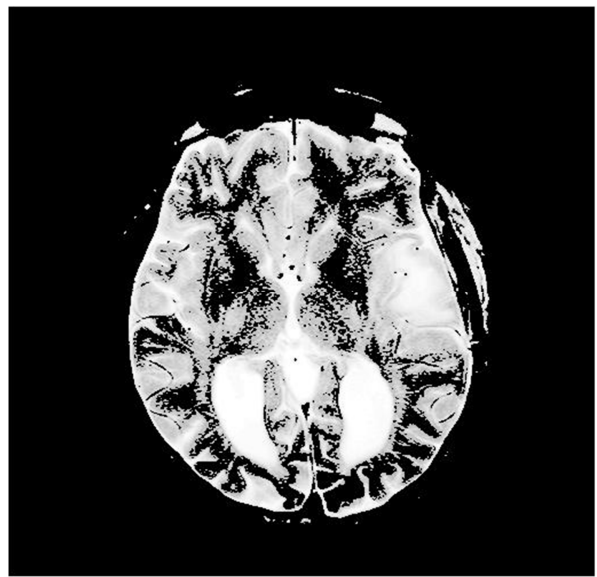


Fig. 9. Skull Removed.

Segmented Image

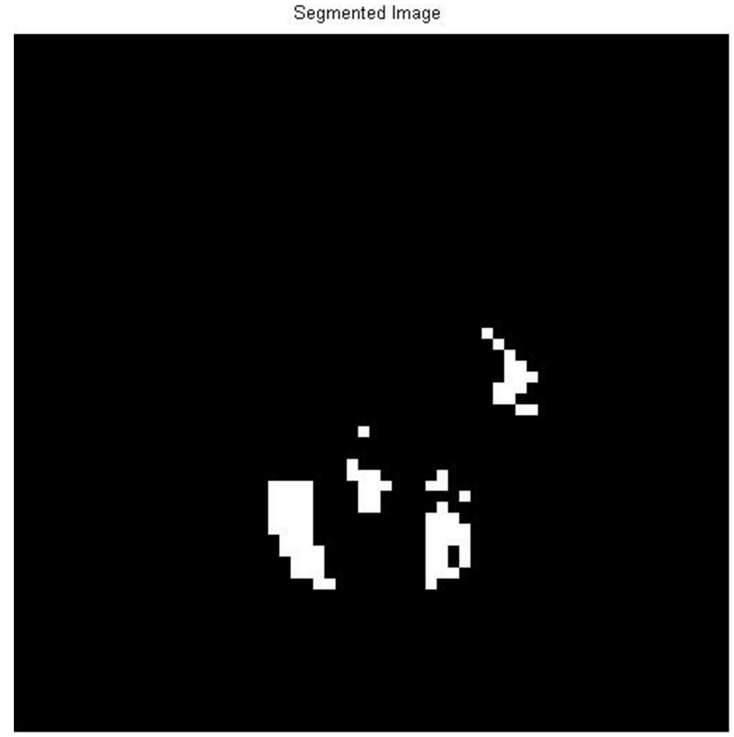

After Morphological Operation

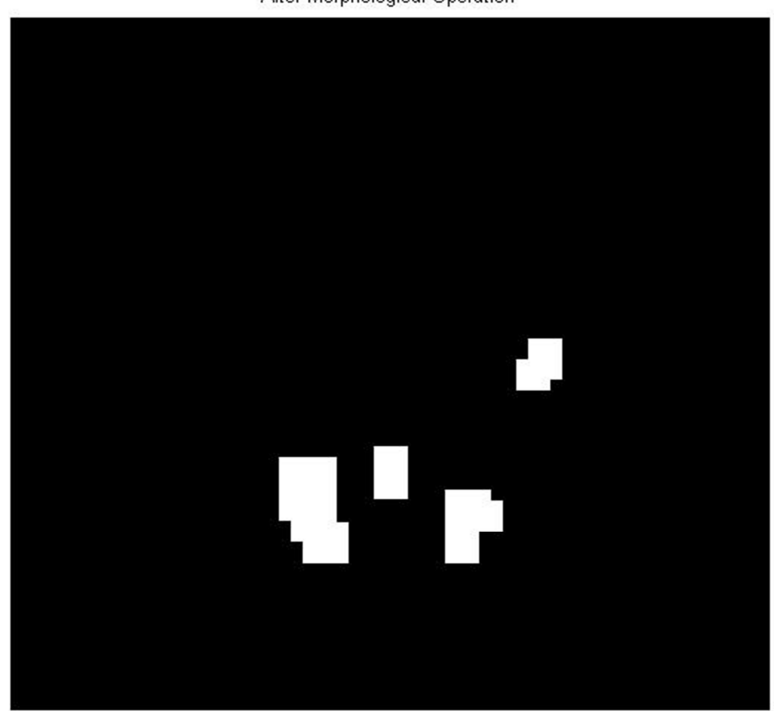

Fig. 11. Tumor.

Fig. 10. Segmented Image.

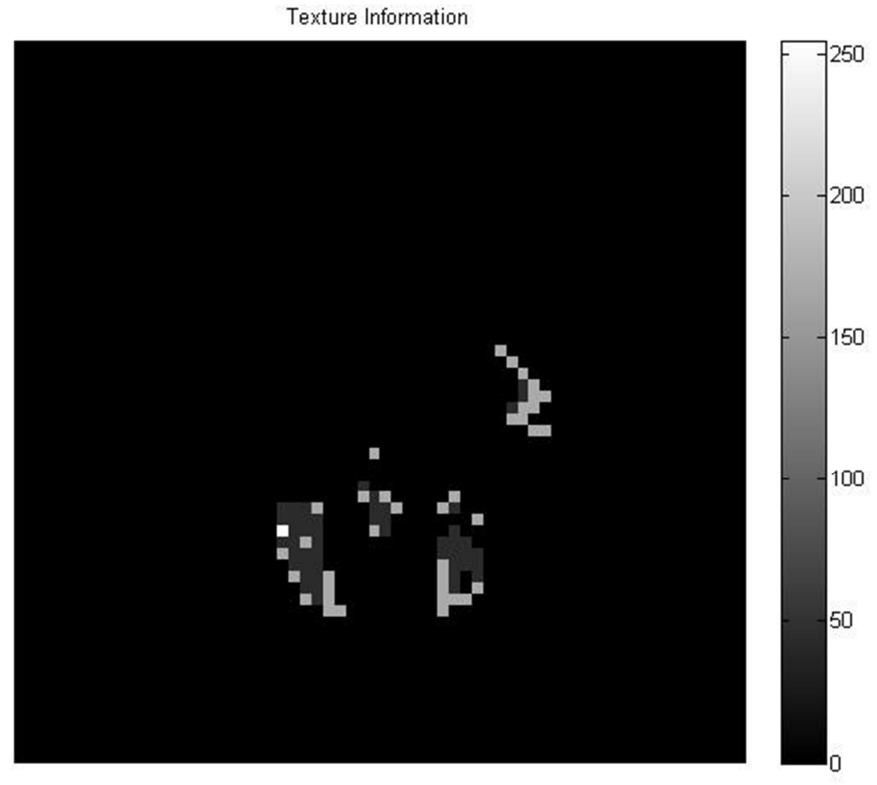

Fig. 12. Texture Detail.

Table 8. Obtained Texture Values for the Tumor.

\begin{tabular}{|c|c|c|c|}
\hline \multicolumn{2}{|c|}{$\begin{array}{l}\text { Smooth Texture of Brain Tumor Surface with } 100 \% \text { Smoothness and } \\
0 \% \text { Roughness }\end{array}$} & \multicolumn{2}{|c|}{$\begin{array}{l}\text { Rough Texture of Brain Tumor Edge with } 60 \% \text { Smoothness and } 40 \% \\
\text { Roughness }\end{array}$} \\
\hline Feature values & Values & Feature values & Values \\
\hline Mean Level 1 & 1.45772585 & Mean Level 1 & 1.34633022 \\
\hline Area Level 1 & 239.1263369 & Area Level 1 & 235.9642857 \\
\hline Dimension Level 1 & 1496 & Dimension Level 1 & 392 \\
\hline Mean Level 2 & 1.498535377 & Mean Level 2 & 1.761117175 \\
\hline Area Level 2 & 238.7133867 & Area Level 2 & 233.3372549 \\
\hline Haar Average Level 1 & 99.99902423 & Haar Average Level 1 & 92.99393589 \\
\hline Haar Detail Level 1 & 2.459757713 & Haar Detail Level 1 & 6.064112307 \\
\hline Daubechies Average Level 1 & 98.74385556 & Daubechies Average Level 1 & 95.99413996 \\
\hline Daubechies Detail Level 1 & 0.095614444 & Daubechies Detail Level 1 & 5.006003782 \\
\hline Bi-Orthogonal Average Level 1 & 99.99901163 & Bi-Orthogonal Average Level 1 & 92.99392059 \\
\hline Bi-Orthogonal Detail Level 1 & 2.698836768 & Bi-Orthogonal Detail Level 1 & 6.079409778 \\
\hline Coiflet Average Level 1 & 98.45903827 & Coiflet Average Level 1 & 96.99443848 \\
\hline
\end{tabular}


So from the Texture detail image [Fig. 12] it can be seen that the tumor boundaries are slight rough whereas the center and rest portion are having very smooth textures.

Gray Scale Values corresponding to different classes:

Table 9. Gray Level Indicating Different Classes.

\begin{tabular}{ll}
\hline Class & $\begin{array}{l}\text { Gray Scale Value } \\
\text { (Black -> 0 \& } \\
\text { White-> 255) }\end{array}$ \\
\hline Very Smooth (0\% Rough 100\% Smooth) & 42.5 \\
Moderately Smooth (20\% Rough 80\% Smooth) & 85 \\
Slight Smooth (40\% Rough 60\% Smooth) & 127.5 \\
Slight Rough (60\% Rough 40\% Smooth) & 170 \\
Moderately Rough (80\% Rough 20\% Smooth) & 212.5 \\
Very Rough (100\% Rough 0\% Smooth) & 255 \\
\hline
\end{tabular}

\section{Conclusion}

The proposed technology is useful to doctors as well as to the diagnostic center as it portrays the texture that tumor is having. Our algorithm defines the texture of tumor in reference to our daily known textures. The proposed state-ofthe-art algorithm utilizes both fractal analysis along with wavelet features to make the prediction much more firm in terms of accuracy. Generally, tumor appears as very smooth type and near the sides it becomes slight rough. The texture detail image will let medical practitioner know the type of texture of the tumor. This will help in diagnostics of the Brain tumor by correlating with other clinical tests and findings.

\section{References}

[1] W. M. Smeulders, M. Worring, S. Santini, A. Gupta, and R. Jain, "Content-based image retrieval at the end of the early years," IEEE Trans. Pattern Anal. Mach. Intell. 22 (12), pp. 1349-1380, 2000.

[2] Mind the Gap: Another look at the problem of the semantic gap in image retrieval Jonathon S. Harea, Paul H. Lewisa, Peter G. B. Enserb and Christine J. Sandomb School of Electronics and Computer Science, University of Southampton, UK; School of Computing, Mathematical and Information Sciences, University of Brighton, UK.
[3] An Efficient Algorithm for Fractal Analysis of Textures, Alceu Ferraz Costa, Gabriel Humpire-Mamani, Agma Juci Machado Traina, Department of Computer Science, University of São Paulo, USP, São Carlos, Brazil \{alceufc, ghumpire, agma\}.

[4] Filterbanks and transforms Sources: Zölzer, "Digital audio signal processing," Wiley \& Sons. Saramäki, "Multirate signal processing," TUT course.

[5] Multiscale Wiener filter for the restoration of fractal signals: wavelet filter bank approach, Bor Sen Chen, Dept. of Electr. Eng., Nat. Tsing Hua Univ., Hsinchu, Taiwan, Chin-Wei Lin.

[6] B. Mandelbrot, "Some noises with 1/f spectrum", IEEE Trans. Inform. Theory, vol. IT-13, pp. 289-298, 1967.

[7] A Computer Algorithm for Determining the Hausdorff Dimension of Certain Fractals By Lucy Garnett, mathematics of computation volume 51, number 183 july 1988, pages 291300 .

[8] B. MANDELBROT, The Fractal Geometry of Nature, Freeman, San Francisco, Calif., 1983.

[9] Fast fractal stack: fractal analysis of computed tomography scans of the lung. Alceu Ferraz Costa, Joe Tekli and Agma Juci Machado Traina, International ACM Workshop on Medical Multimedia Analysis and Retrieval (MMAR), pp. 1318, 2011.

[10] A k-nearest neighbor based algorithm for multi-label classification, Min-Ling Zhang, National Lab. for Novel Software Technol., Nanjing Univ., China Zhi-Hua Zhou

[11] D. W. Aha, "Special Al review issue on lazy learning", Artificial Intelligence Review, vol. 11, 1997.

[12] A Fast Algorithm for Multilevel Thresholding, PING-SUNG LIAO, Department of Electrical Engineering chengshiu Institute of Technology Kaohsiung, 833 Taiwan TSE-SHENG CHEN, Department of Engineering Science National Cheng Kung University Taiwan 701 Taiwan, AND PAU-CHOO CHUNG, Department of Electrical Engineering National Cheng Kung University Tainan, 701 Taiwan.

[13] S. U. Lee and S. Y. Chung, "A comparative performance study of several global thresholding techniques for segmentation," Computer Vision Graphics Image Processing, Vol. 52, 1990, pp. 171-190. 